\title{
Ford Escape PHEV On-Road Results from US DOE's Technology Acceleration and Deployment Activity
}

\author{
Richard “Barney” Carlson ${ }^{1}$, Julie D’Annunzio ${ }^{2}$, Christopher Fortin ${ }^{2}$, Matthew Shirk ${ }^{1}$ \\ ${ }^{1}$ Idaho National Laboratory, ${ }^{2}$ Ford Motor Company
}

\begin{abstract}
The Idaho National Laboratory (INL) conducts fleet data collection and analysis activities for advanced technology vehicles, including Plug-In Hybrid Electric Vehicles (PHEV), as part of the US DOE's Advanced Vehicle Testing Activity (AVTA). In 2009, data collection began for the Technology Acceleration and Deployment Activity (TADA) for which INL is conducting the data analysis and reporting. The Technology Acceleration and Deployment Activity includes twenty-one Ford Escape PHEVs operated by various fleets throughout the world. Data is collected from all twenty-one vehicles during driving and charging in order to understand the operation of the vehicle as well as the vehicle operator behavior.
\end{abstract}

This paper discusses the overall results from a three year period of data collection from the twenty-one E85 capable Ford Escape PHEVs, which amounts to over 500,000 miles of operation. Multiple factors that impact the fuel economy and electrical energy consumption are presented including charging behavior, ambient temperature, driving style, accessory usage, and route type.

Keywords: PHEV, fleet, lithium battery, energy consumption, efficiency

\section{Introduction}

The Ford Escape Plug-In Hybrid Electric Vehicle (PHEV) Advanced Research project was established to help identify a sustainable pathway toward the accelerated and successful mass production of PHEVs. Through proactive demonstration of their electrification technology, Ford sought to encourage open collaboration on the development and delivery of PHEV/Grid solutions.

Ford began the project in 2007 in partnership with Southern California Edison. In 2008 the project was expanded to include the Electric Power Research Institute (EPRI) and 10 additional utilities (National Grid, NYSERDA, New York Power Authority, American Electric Power, ConEdison, Progress Energy, PEPCO, Southern Company, Hydro Quebec, and DTE Energy). Also in 2008, the DOE awarded its support to the project through both the approval of a funding award and by participation in the vehicle evaluations. The DOE is presently evaluating three of the twenty-one deployed Escape PHEVs (figure 1). As of the writing of this paper, the remaining eighteen Escape PHEVs are currently deployed with utilities and Ford Engineering across the US, Canada, the UK and China.

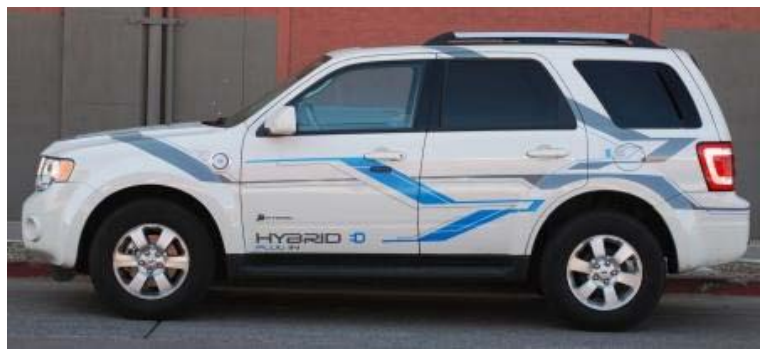

Figure 1: Ford Escape Hybrid Plug-in

The Escape PHEVs of the research fleet are modified Escape Hybrids. The production batteries were removed and replaced with high capacity 
lithium ion batteries capable of being charged externally. These modifications allow the vehicle to operate in the electric-propulsion charge depleting (CD) mode, minimizing the use of the ICE to peripherals and power assists. Once the high voltage battery has roughly one-third charge remaining, the vehicle transitions to charge sustaining (CS) mode, operating as a standard hybrid. Some engines were also updated with Flex Fuel (E85) hardware and software. The purpose of the PHEV update was to demonstrate vehicle/grid interaction and customer duty cycles; vehicles were not optimized to provide maximum potential fuel economy.

Under the DOE's guidance, Ford has been working with Idaho National Laboratory (INL) to conduct fleet data collection and evaluation for reporting on the Advanced Vehicle Testing Activity (AVTA) website ${ }^{1}$. INL and Ford have worked together to identify data collection parameters, analysis metrics, and reporting methods. Ford created an automated process and software to provide vehicle data to INL. Ford and INL have completed their data validation analysis and reports have been published on a monthly basis since November 2009. Since the start of the project, over 500,000 miles of driving data have been accumulated and analyzed on a trip-by-trip basis.

\section{Data and Summary Reports}

\subsection{Data Collection}

Each of the Ford Escape PHEVs in operation is equipped with a data collection system. Figure 2 shows the data collection and transfer process. Vehicle data is collected continuously and stored within the data collection device (1) for transmission through the broadband wireless network (2) which relays the data to be archived in a collection server (3). The data is then relayed to a website server (4) where it is available to authorized users through the website server (5/6). The Escape PHEV website is capable of processing incoming data on a second-by-second basis to allow for the creation of reports in real time. These reports include both overall fleet and individual vehicle reports. The fleet has experienced some periodic interruptions in the transmission and receipt of vehicle data primarily due to intermittent broadband wireless accessibility. This has occurred when the vehicle has been out of broadband range (i.e., when the vehicle is parked in underground parking structures), there has been a fault within the vehicle broadband modem or the wireless provider has experienced a service issue. During all of these occurrences the data has remained stored within the vehicles data acquisition system ready for transmission once the broadband connection is reestablished.

1. Data collected onvehicle.

2. Data received by broadband wireless network

3. Data archived in collection server

4. Data relayed to website server

5. Website server backed-up nightly

6. Data available to authorized users through web

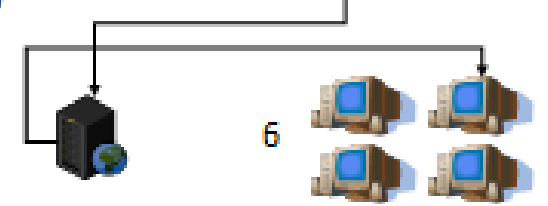

Figure 2: Diagram of data collection and storage process from Ford Escape PHEV fleet 


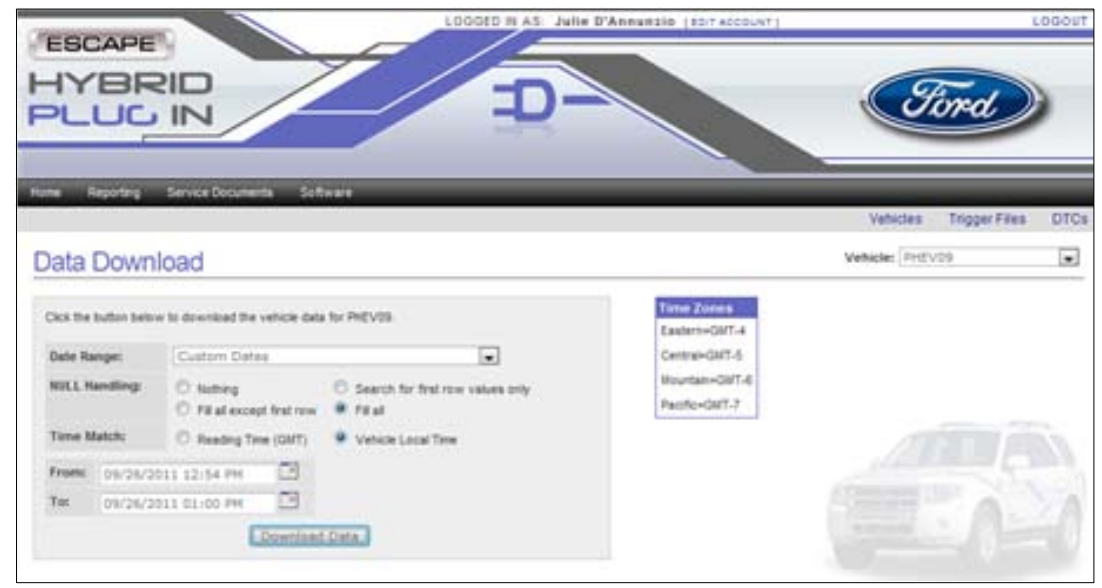

Figure 3: Vehicle operator user interface for website data download

\subsection{Raw Data Collected}

Vehicle driving and charging data is collected on over 50 parameters from the battery, powertrain, charger, and vehicle chassis controllers. In the case of a field issue or service concern, this data can be accessed to determine what the vehicle was experiencing at the time of the occurrence. Second-by-second data is available for each vehicle from the web server. The website interface is shown in figure 3.

\subsection{Drive and Charge Summary Data}

Engineering reports, available to a password authenticated user, compile the second-bysecond data into summary data collected from each event. Data is available in a summarized report for each individual Escape PHEV drive for the specific date and time range requested. The drive summary data available on the Escape PHEV website includes parameters such as average speed, battery temperature, electrical energy consumption, fuel consumed, distanced travelled, GPS location, and other vehicular information. Charge event data is also available in a summarized report for each individual Escape PHEV. The charge summary data includes wall energy to charger, energy that enters the battery, battery temperature, ambient temperature, and many more parameters.

\subsection{Fleet Performance Reports}

As part of this collaboration between Ford Motor Company and the DOE's AVTA, monthly reports are produced by INL summarizing the month's performance of the fleet of Escape PHEVs. These reports summarize vehicle usage parameters, including distance traveled; fuel economy, electrical energy consumption, and timeof-day charging. Also included in these reports are route type, and ambient operating conditions. The AVTA website is available at http://avt.inl.gov.

In addition to the INL fleet summary reports, all project partners have access to the data that is being collected via the on-board data acquisition system. Depending upon the vehicle's access to broadband wireless this provides near real time data availability. Partners have access to performance reports for individual fleet vehicles in the field as well as for the entire fleet. Reports can be filtered to select desired date, time, and parameters.

\section{Analysis and Discussion}

\subsection{Overall Energy Consumption}

PHEVs typically have two modes of operation. Charge Depleting mode (CD) entails propulsion energy coming from electrical energy from onboard energy storage whereas Charge Sustaining mode (CS) ultimately derives all propulsion energy from liquid fuel. In $\mathrm{CD}$ operation the internal combustion engine may or may not be used, as designed by the vehicle manufacturer. If the engine is not utilized in CD operation, then it is considered to be all electric operation. If the internal combustion engine is utilized, it is known as blended operation.

The Ford Escape PHEV primarily operates in a blended mode of operation when in (CD) mode. The engine may operate during $\mathrm{CD}$ mode as necessary but at significantly reduced amounts of time and power levels. A large portion of the propulsion energy is from electrical energy stored in the on-board battery system. Using electrical 
propulsion energy inherently improves gasoline fuel economy in CD mode as compared to CS mode. Some driving trips may begin in CD mode but end in CS mode (CD/CS) when the usable propulsion energy from the battery system is depleted during driving. These trips, on average, show some of the fuel economy benefits of the CD operation.

INL conducted baseline fuel economy testing over the EPA standard urban drive (UDDS) and highway (HWFET) drive cycles on one Escape PHEV using E-85 fuel ${ }^{2}$. Figure 4 shows the baseline UDDS CD and CS fuel economies established.
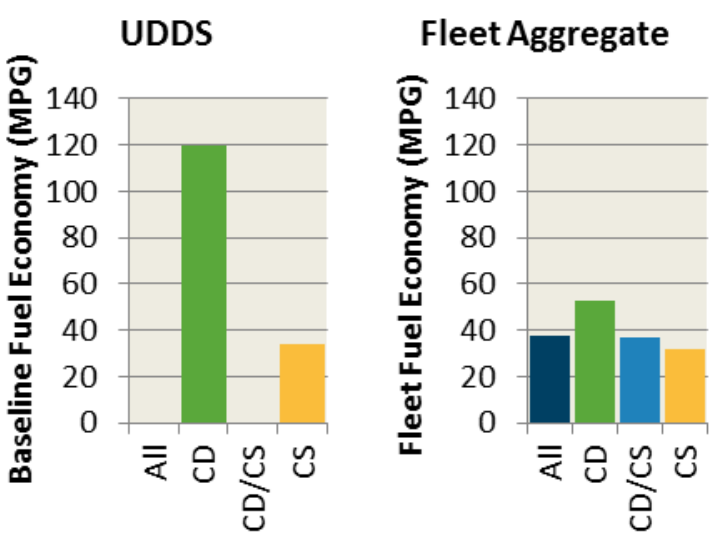

Figure 4: Baseline and Fleet Fuel Economy (All and $\mathrm{CD} / \mathrm{CS}$ not evaluated in baseline tests)

Also shown in figure 4 are the fuel economies of the entire fleet for November 2009 - July 2011. A comparison of the results shows significantly higher fuel economy results achieved in baseline testing versus the infield fleet despite the fact that many of the fleet vehicles in the field used regular gasoline (Note: E-85 fuel economy results are typically $20-25 \%$ lower than standard gasoline due to the decreased energy content on a volumetric basis in E85 versus gasoline).

Factors contributing to fuel economy performance in the field can be grouped into two general categories: reduction of CD operation and general energy consumption increase. Factors affecting charge depletion operation are discussed in section 3.2; the remaining energy consumption factors are discussed in sections 3.3-3.5.

\subsection{Overall Fleet Driving and Charging Characteristics}

The Ford Escape PHEVs in this study are primarily being driven in fleet operations by electric utility companies. A review of the vehicle data collected, including both driving and charging patterns, indicates that the vehicles are being operated in a manner different than what is anticipated for typical personal use PHEVs. The vehicles in this study are being driven roughly every other day for an average daily distance of 56 miles. They are being charged roughly three times per week. This is different than the expected typical personal use of driving 30 miles per day in combination with one plug-in per day ${ }^{3}$. In addition, the city and highway driving characteristics being experienced by this fleet are different from that expected for typical private vehicles. The industry standard for route type (city or highway driving) is based off of historical data which indicates $55 \%$ city and $45 \%$ highway driving with an average vehicle speed separation between city and highway trips of $42 \mathrm{mph}^{3}$. Overall the Escape PHEV fleet drives at slightly higher speeds than the historical average as shown in figure 5 , since only $45 \%$ of the trips are considered to be city trips ( $\leq 42 \mathrm{mph})$ as compared to $55 \%$ for historical average. CD trips for the Escape PHEV fleet are predominantly city trips since $80 \%$ of miles are less than or equal to $42 \mathrm{mph}$. These vehicle usage differences directly impact fuel economy and electrical energy consumption since the overall vehicle efficiency differs from city to highway operation.

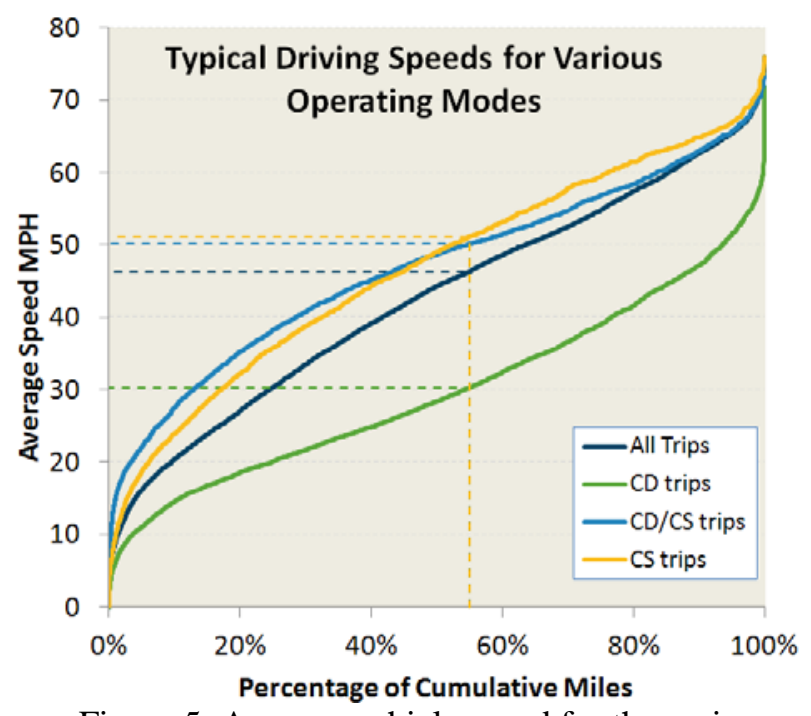

Figure 5: Average vehicle speed for the various operating modes of the vehicle 
A closer look at the Escape PHEV fleet driving patterns indicate that short trips ( $<40$ miles) provide the bulk of the fleet's mileage (59\%). While more than half of the fleet's mileage comes from short trips, a significant portion (14\%) of the mileage is provided by trips longer than 100 miles, facilitated by the vehicles' gasoline engine. For the shorter trips, more than half of the mileage is accumulated in CD mode, but with longer trips this CD trend is reversed with almost all the mileage accumulated in CS mode (Figure 6). This occurs because the onboard battery becomes depleted during these longer trips, triggering the mode change.

A significant portion of trips contain both $C D$ and CS driving. Trips which contain both modes $(\mathrm{CD} / \mathrm{CS})$ are shown in figure 6 with their contributions to CD and CS mileages indicated via slashed bars. The presence of $C D$ mileage at ranges above the maximum CD range of 40 miles is due to longer trips starting off in CD mode depleting the battery past the mode transition threshold and then transitioning to CS mode. Overall CS mode accounts for 58\% of all mileage, including $\mathrm{CD} / \mathrm{CS}$ trips. Purely CS trips contribute $31 \%$ of the fleet's mileage, CD/CS trips $43 \%$, and purely CD trips $26 \%$. The large percentage of CS mileage (58\%) experienced by the fleet is a significant contributor to the overall realized fleet fuel economy.

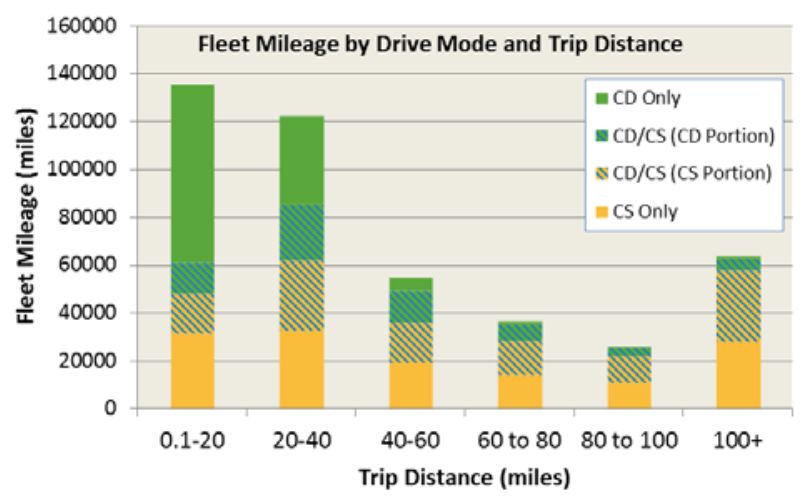

Figure 6: Fleet Mileage by Drive Mode and Trip Distance

A look at the charging patterns being experienced by the fleet helps explain the large percentage of CS mileage since the drive mode of the vehicles is determined by the state of charge (SOC) of the on-board High Voltage (HV) battery. Figure 7 indicates that the average HV battery SOC at vehicle start-up is $57 \%$.

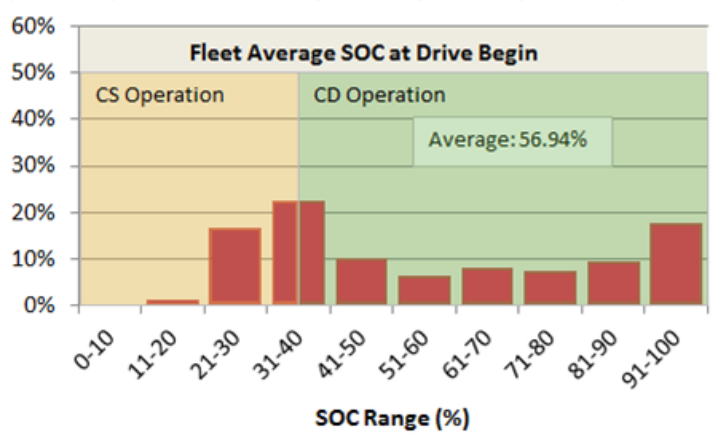

Figure 7: Fleet Average SOC at Drive Begin

Only $18 \%$ of the time do the fleet drives start with a full charge ( $90 \%$ or greater SOC). In addition, more than $40 \%$ of all trips begin with $40 \%$ SOC or less, giving minimal or zero $\mathrm{CD}$ range. This pattern of beginning drives with low HV battery SOC severely limits the ability to realize potential benefits of the PHEV system.

A closer look at the fleet charging data reveals that the fleet vehicles leave charge events with an average SOC of $81 \%$, and a full charge $(>90 \%$ SOC) only $55 \%$ of the time. This means almost half of all charge events are interrupted before they are complete.

When the Escape PHEV fleet was designed, the anticipated PHEV charging pattern was one complete charge taking place overnight. In practice however, the fleet sees the opposite of this behavior, with $75 \%$ of all charging taking place between $7 \mathrm{AM}$ and $6 \mathrm{PM}$ as shown in figure 8 .

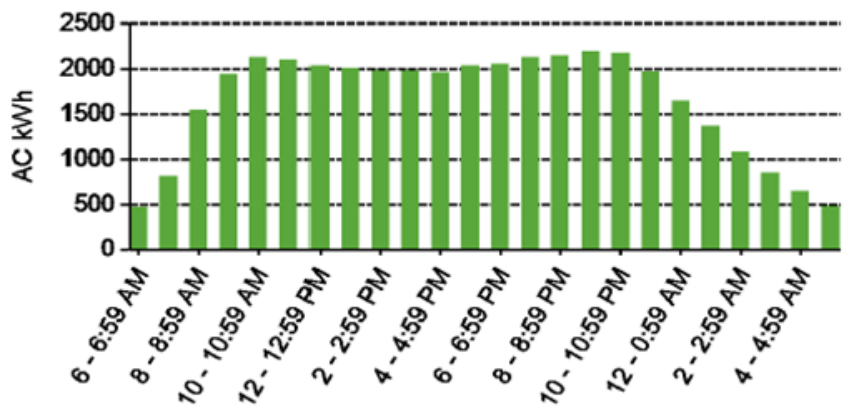

Figure 8: Time of Day when Charging

In addition the vehicle is charged roughly three times per week with the charge duration typically lasting slightly less than 2 hours and increasing the battery's SOC by only 27\% (Figure 9). Due to these short, infrequent charges, the vehicles routinely operate with low SOC, forcing the fleet into CS mode much more often than initially projected. This charging behavior has a far- 
reaching impact on the vehicles' operating mode and fuel economy.

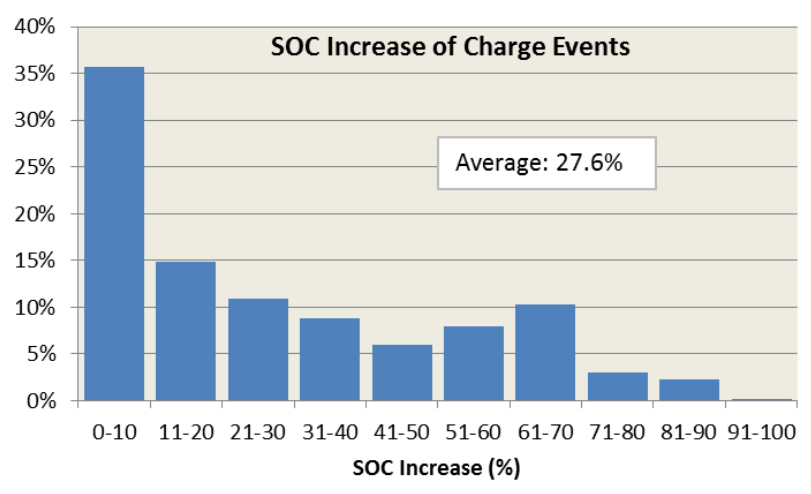

Figure 9: SOC Increase of Charge Events

The Escape PHEV was designed to have a 40 mile CD range when starting with a full SOC, and then transition to CS mode. As indicated in figure 10, roughly two thirds of all charge events have 40 miles or less accumulated before recharging. Recalling the previously discussed drive and charge patterns, if these incomplete charge events were allowed to finish and reach full SOC (e.g. overnight), CD operation would drastically increase which would directly increase the overall fuel economy of the vehicle by merely utilizing more electrical energy.

The important role that charging plays in capitalizing on a PHEV's potential is depicted in Figure 11, which shows the overall trend between vehicle fuel consumption and drive event distance in combination with the starting SOC of the drive event. Regular and full charging allows for higher high-voltage battery SOC values at the beginning of a drive. A vehicle which starts off with a full charge typically requires less liquid fuel over the drive than those which start out at lower SOC values. The initial dip shown in all drive events, including those with high beginning SOC values, occurs because even while operating in CD mode, certain systems require the engine to be engaged at drive start. This inflates the use of gasoline for these short trips. Overall, the maximum benefit of charging the HV battery is realized in the 10 to 40 mile trip range. As the trip length increases, the benefits trend toward standard HEV (non-PHEV) operation.

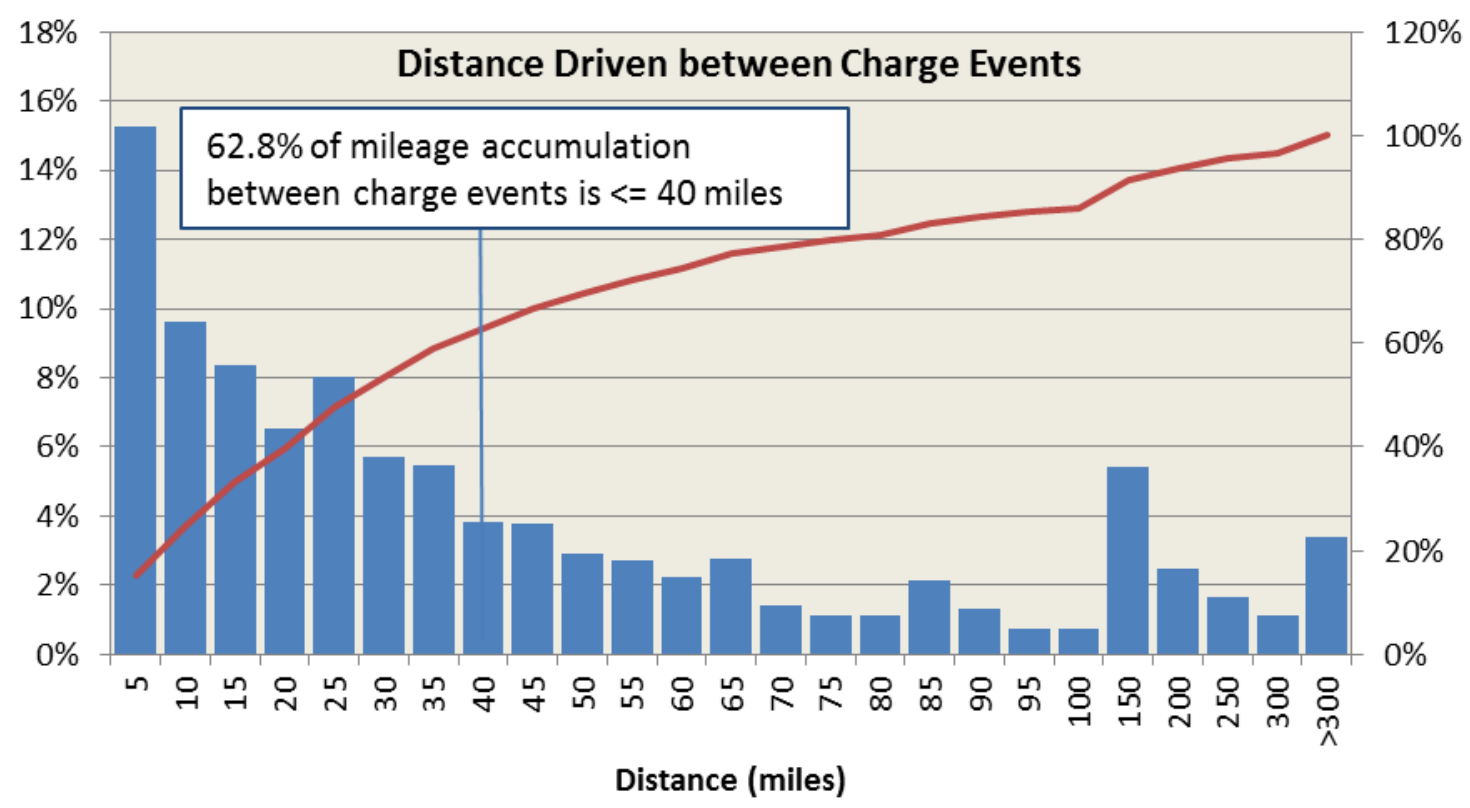

Figure 10: Distance Driven between Charge Events 


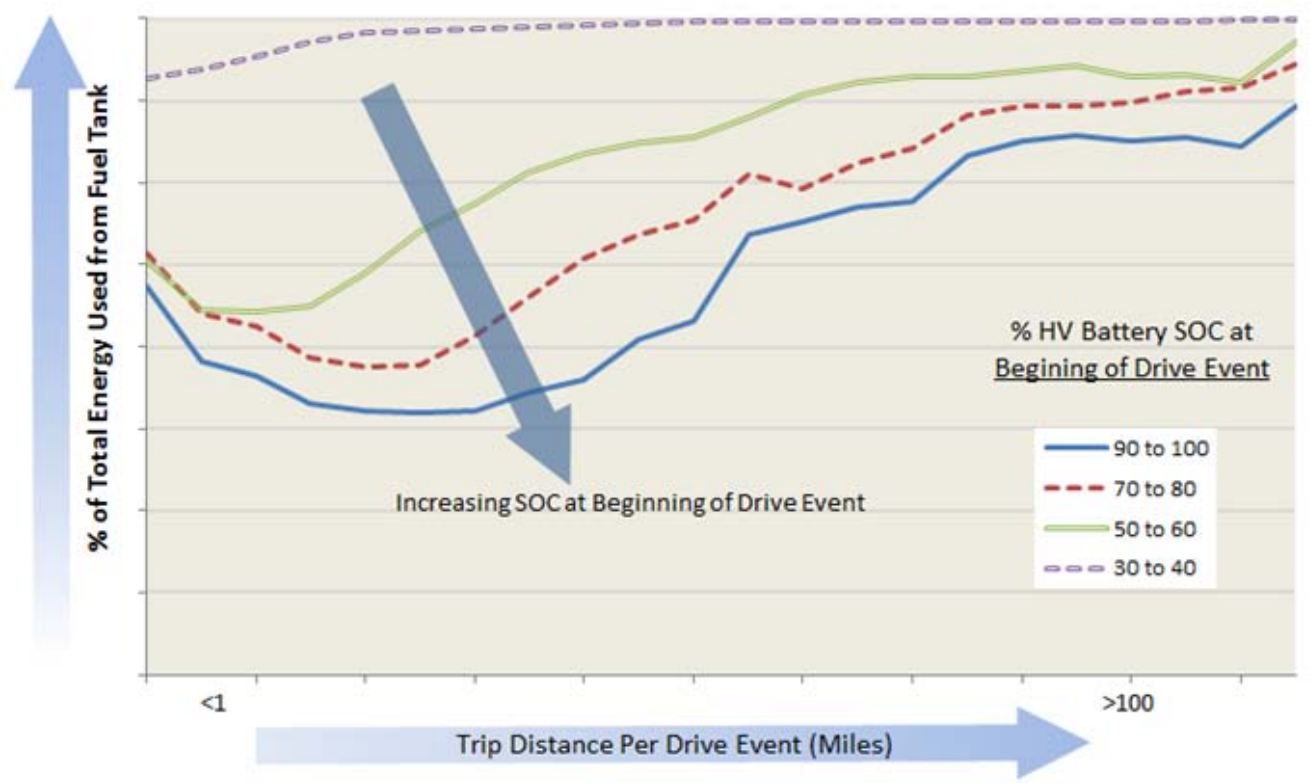

Figure 11: Impact of Starting HV Battery SOC: Percent of Trip Energy from Fuel per Drive Event Distance

\subsection{Ambient Temperature Impact on Fuel Economy}

Ambient temperature has been determined to significantly impact fuel economy and electrical energy consumption due to multiple factors. These factors include engine operation for cabin heat, air conditioner operation, driveline friction variation due to fluid viscosity and material property changes, and decreased battery power and energy capabilities at extreme temperatures. Figure 12 shows the combined effects of ambient temperature on fuel economy for each operating mode.

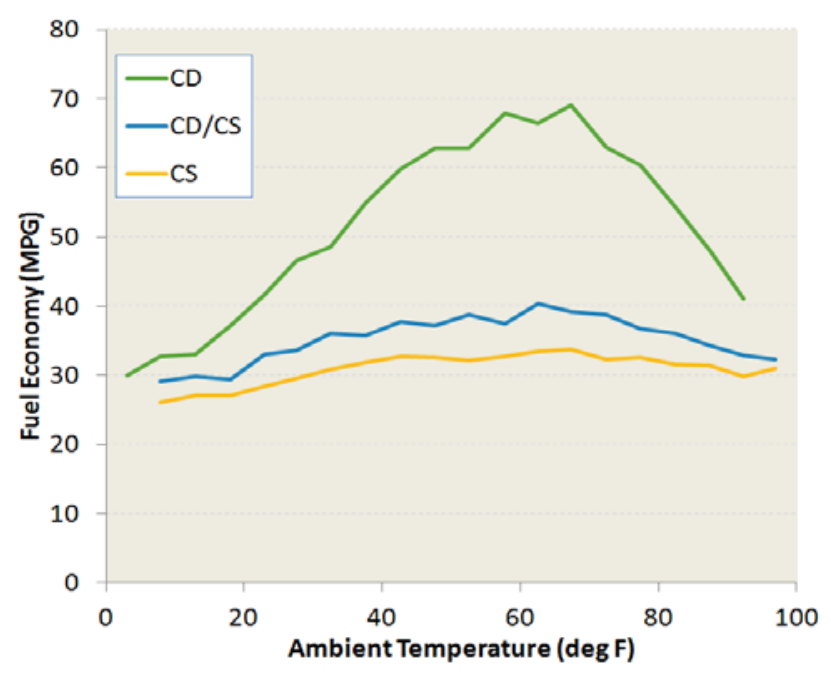

Figure 12: Ambient Temperature impact on Fuel Economy

The trend shows CD fuel economy significantly increases compared to CS fuel economy in moderate temperate conditions (40 to 80 degree $\mathrm{F}$ ) to the extent of twice the fuel economy near 70 degree $\mathrm{F}$ as compared to CS operation.

\subsection{Air Conditioner Utilization}

At high ambient temperatures, the air conditioner system is operated more often and at higher power levels which increases overall energy consumption of the vehicle since the air conditioner system is consuming fuel energy in addition to the vehicle propulsion energy requirements. Additionally the air conditioner is belt driven by the engine which requires the engine to operate whenever the air conditioner compressor is operating. Figure 13 shows the percent of miles travelled with the air conditioner system operating as a function of ambient temperature. As expected the air conditioner operates a moderate amount at temperatures between freezing and 60 degrees $\mathrm{F}$ for use with windshield defrost. But as ambient temperature continues to increase, the utilization of air conditioning system dramatically increases. 


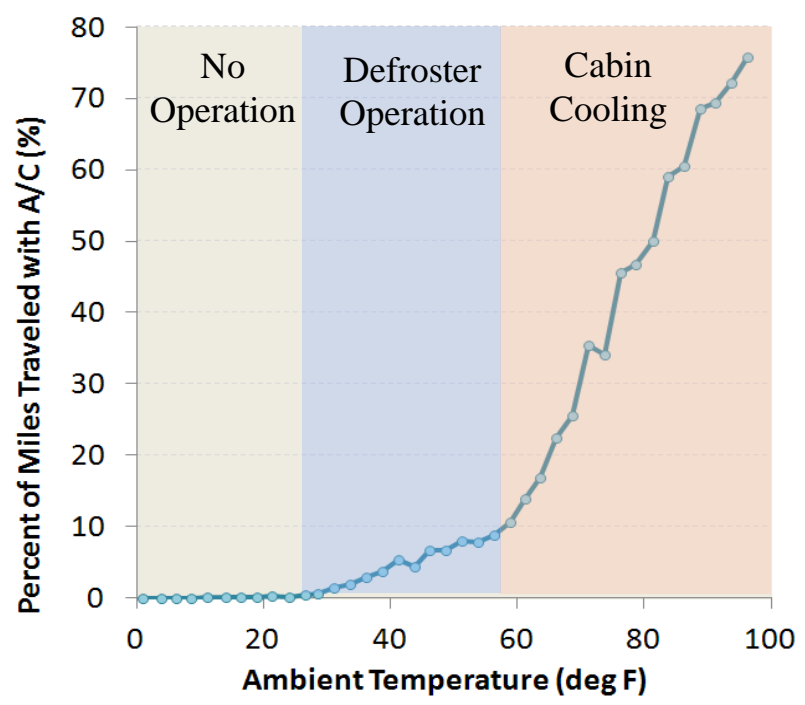

Figure 13: Percent of Miles travelled with Air Conditioner operating

To isolate the impact of the air conditioning system from the other ambient temperature effects on fuel economy, trips with no air conditioner operation are isolated and the fuel economy is determined for each operating mode. This is shown on figure 14 for comparison to the fuel economy of trips which includes all ambient temperature effects as previously shown in figure 12 . Note for the trips with no air conditioner utilization, the fuel economy significantly increases at high ambient temperatures. This is due to a large amount of electric propulsion energy resulting in higher electrical consumption (DC Wh/mi).

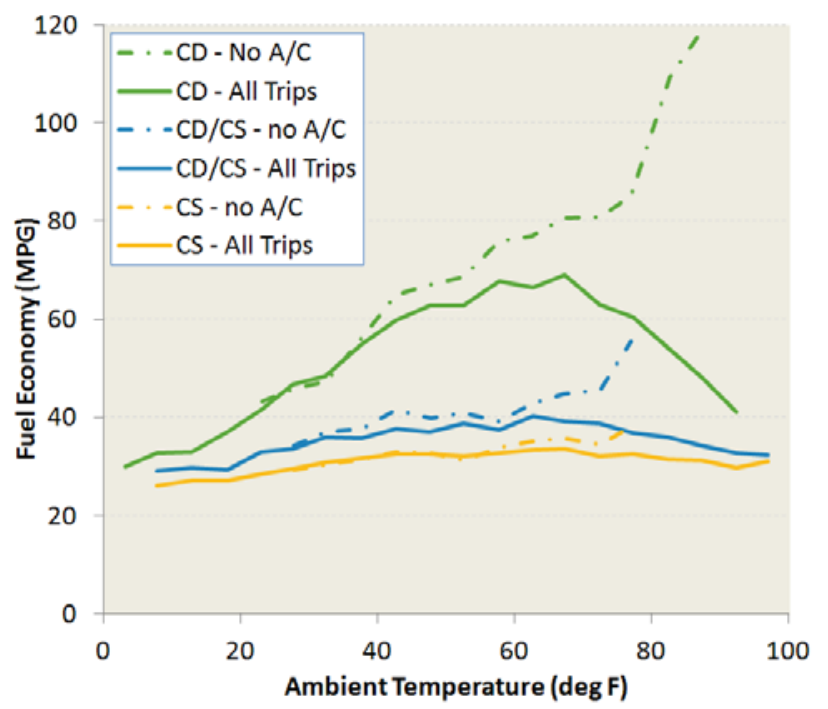

Figure 14: Air Conditioner operation effect on Fuel Economy

\subsection{Driving Style Impact on Energy Consumption}

There are many metrics than can be used to quantify the style in which the vehicle is operated by the driver (driving style) but for the simplification of this discussion, driving style will simply be a linear metric from one to ten where one is a very energy conscious driver operating the vehicle at low speeds and low acceleration rates. Conversely a driving style of ten is a very quick responding, almost aggressive style including very high acceleration rates and high speed driving. The driving style only describes the speed and acceleration patterns which are independent from the other factors previously discussed such as A/C operation, ambient temperature, and charging patterns.

Driving style has been shown to influence the fuel economy and electrical energy consumption of PHEVs ${ }^{4}$. Through analysis of the data collected from the Ford Escape PHEV fleet, driving style has been shown to impact fuel economy and electrical energy consumption due to its significant all electric operation capability with 'efficiency conscious' driving style (low driving style number) when the battery pack is charged. Conversely, at high driving style numbers (high power driving demands, high acceleration and high speed driving), the engine is used to supplement the electrical propulsion system even when the battery pack is charged. This impacts fuel economy by increasing liquid fuel consumption. Figure 15 shows the percent of miles driven in all electric operation with respect to driving style. With increasingly higher driving style, the percentage of miles in all electric operation decreases significantly.

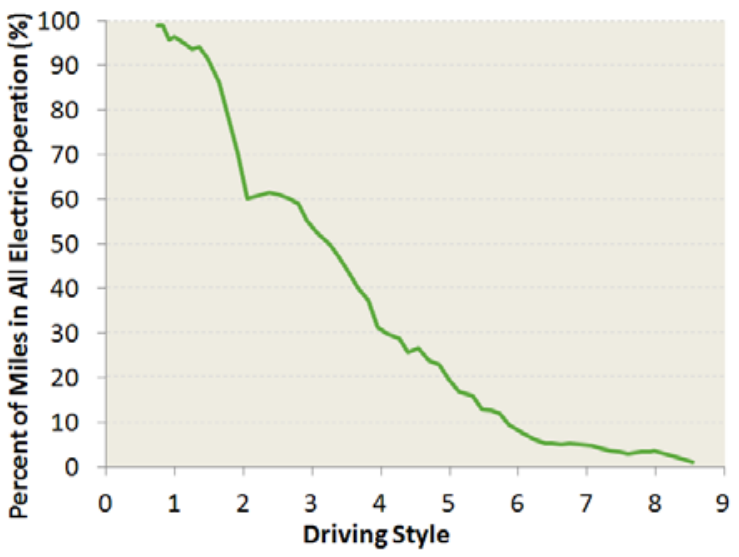

Figure 15: All Electric operation decreases with increasing Driving Style (only CD trips shown) 
Operating mode (CD or CS) is dependent on the amount of usable electrical energy stored in the battery pack and is therefore independent from driving style. Analysis was performed to determine if driving style impacts fuel economy in both CD and CS operation and to the same magnitude. The results of this analysis show driving style generally impacts the fuel consumption in the same manner for both CD and CS. This increase in fuel consumption for CD and CS modes with increasing driving style is shown in Figure 16. Additionally, this figure shows the fuel consumption difference between CD and CS (difference between the two lines) which is the petroleum displacement gained from CD operation.

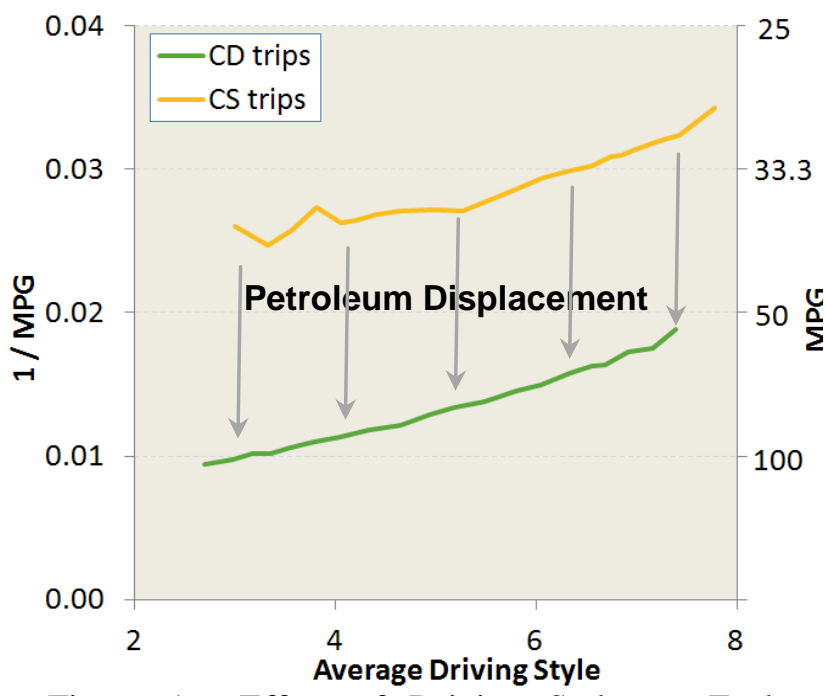

Figure 16: Effect of Driving Style on Fuel Consumption for CD and CS operation

\section{Summary}

The Technology Acceleration and Deployment Activity vehicle demonstration project has shown continued petroleum reduction through advances in vehicle technologies. This is demonstrated by the data, analysis and results from over 500,000 miles of operation of twenty-one Ford Escape Hybrid Plug-ins over a three year period as well as baseline vehicle testing over EPA drive cycles. While baseline testing defines the PHEV hardware and software capabilities, other factors need to be considered to enable the customer to take full advantage of this technology and optimize their vehicle's performance. Driving and charging patterns have been identified as impacting energy consumption and underscore the need for infrastructure upgrades to enable regular and frequent charging. In addition, driving style, ambient temperature, available onboard electrical energy, route type, and accessory usage have been identified as substantially influencing energy consumption and fuel economy. The Escape PHEV advanced research fleet delivers the best fuel economy with frequent and complete battery charging, mid-range trips, and moderate operating temperatures. When assessing the impact of PHEVs on fuel dependence, factors which maximize CD mode operation and minimize ancillary energy consumption need to be taken into consideration.

\section{References}

[1] The Advanced Vehicle Testing Activity website, Idaho National Laboratory, http://avt.inel.gov/phev.shtml

[2] 2010 Ford Escape Advanced Research Vehicle Baseline Performance Fact Sheet, Idaho National Laboratory, 2011

[3] SAE J2841 Sep. 2010 Utility Factor Definitions for Plug-In Hybrid Electric Vehicles Using Travel Survey Data

[4] Carlson, Shirk, et. al.; Factors Affecting the Fuel Consumption of Plug-In Hybrid Electric Vehicles; EVS25; 2010

\section{Authors}

Richard "Barney" Carlson is a research engineer with the Idaho National Laboratory and has been developing, testing, and analyzing Plug-in Hybrid Electric Vehicles since 1994. He is currently working on multiple fleet data collection and analysis projects approaching a total of ten thousand Grid Connected Vehicles and EVSE.

Julie D'Annunzio is the Global Electrified Fleet and Integration Manager at Ford Motor Company. She is currently responsible for the design, development and deployment of global electrified fleets and the subsequent collection and analysis of the real world usage data collected by these vehicles into production solutions.

Christopher Fortin is an engineer at Ford Motor Company performing data acquisition and analysis for global electric fleets. He is currently completing his Master's at Wayne State University in Alternative Energy Technology.

Matthew Shirk is a research engineer with the Idaho National Laboratory and has been working with advanced Technology Vehicles since 2002. He is currently working on multiple fleet data collection and analysis projects approaching a total of ten thousand Grid Connected Vehicles and EVSE. 